\title{
Erratum to: A Clinical Assessment Tool for Advanced Theory of Mind Performance in 5 to 12 Year Olds
}

\author{
Anne E. O'Hare • Lynne Bremner • \\ Marysia Nash · Francesca Happé • Luisa M. Pettigrew
}

Published online: 20 February 2010

(C) Springer Science+Business Media, LLC 2010

Erratum to: J Autism Dev Disord (2009) 39:916-928

DOI 10.1007/s10803-009-0699-2

The story labeling in Table 1 was incorrect. The correct order now shown below (Table 1), is in keeping with the order of administration and Appendix 2.

The online version of the original article can be found under doi:10.1007/s10803-009-0699-2.

\footnotetext{
A. E. O'Hare $(\bowtie) \cdot$ L. M. Pettigrew

Department of Child Life and Health, University of Edinburgh,

20 Sylvan Place, Edinburgh EH9 1UW, UK

e-mail: aohare@ed.ac.uk

L. Bremner · M. Nash

Royal Hospital for Sick Children, Sciennes Road, Edinburgh

EH9 1LF, UK

F. Happé

Institute of Psychiatry, Kings College, De Crespigny Park,

Denmark Hill, London SE5 8AF, UK

Present Address:

L. M. Pettigrew

Whittington VTS, London, UK
} 
Table 1 Mean scores for individual stories and the total according to age for Question 2M

\begin{tabular}{llllllrrrr}
\hline Story & $5 ; 0-5 ; 11$ & $6 ; 0-6 ; 11$ & $7 ; 0-7 ; 11$ & $8 ; 0-8 ; 11$ & $9 ; 0-9 ; 11$ & $10 ; 0-10 ; 11$ & $11 ; 0-11 ; 11$ & $12 ; 0-12 ; 11$ & $P$ value \\
\hline 1 Lie (Dentist) & 0.75 & 0.83 & 1.44 & 1.43 & 1.33 & 1.57 & 1.45 & 1.29 & 0.004 \\
2 White Lie (Hat) & 0.50 & 1.00 & 1.31 & 1.40 & 1.83 & 1.71 & 1.73 & 1.86 & $<0.001$ \\
3 Misunderstanding (Glove) & 0.00 & 0.43 & 0.69 & 1.00 & 1.17 & 1.57 & 1.23 & 1.43 & $<0.001$ \\
4 Sarcasm (Picnic) & 0.00 & 0.09 & 0.62 & 0.62 & 0.78 & 0.90 & 1.14 & 1.71 & $<0.001$ \\
5 Persuasion (Kittens) & 0.00 & 0.30 & 0.44 & 0.67 & 1.39 & 1.43 & 1.36 & 1.71 & $<0.001$ \\
6 Contrary Emotion (Swings) & 0.67 & 0.48 & 0.75 & 0.52 & 1.56 & 1.19 & 0.95 & 1.43 & 0.002 \\
7 Pretend (Bananas) & 1.00 & 0.61 & 1.50 & 1.05 & 1.56 & 1.52 & 1.59 & 1.86 & $<0.001$ \\
8 Joke (Haircut) & 0.25 & 0.17 & 0.69 & 0.95 & 1.06 & 0.67 & 1.05 & 1.71 & $<0.001$ \\
9 Figure of Speech (Cough) & 0.00 & 0.13 & 0.31 & 0.62 & 1.17 & 0.81 & 1.05 & 1.57 & $<0.001$ \\
10 Double Bluff (Ping-Pong) & 0.00 & 0.48 & 0.56 & 0.62 & 0.67 & 1.14 & 0.55 & 0.86 & 0.006 \\
11 Appearance/Reality (Santa Claus) & 1.00 & 1.00 & 1.19 & 1.24 & 1.39 & 1.48 & 1.50 & 1.57 & 0.001 \\
12 Forget (Doll) & 0.50 & 0.83 & 1.38 & 1.24 & 1.17 & 1.57 & 1.09 & 2.00 & 0.001 \\
Total & 4.67 & 6.35 & 10.88 & 11.65 & 15.06 & 15.57 & 14.68 & 19.00 & $<0.001$ \\
\hline P value is for signi
\end{tabular}

$P$ value is for significance of association with age 